\title{
Mapping the Users' Problem Solving Strategies in the Participatory Design of Visual Analytics Methods
}

\author{
Eva Mayr ${ }^{1}$, Michael Smuc ${ }^{1}$, Hanna Risku ${ }^{1}$, Wolfgang Aigner ${ }^{2}$, Alessio Bertone $^{2}$, \\ Tim Lammarsch ${ }^{2}$, and Silvia Miksch ${ }^{2}$ \\ ${ }^{1}$ Research Center KnowComm, Danube University Krems, \\ Dr. Karl Dorrek Str. 30, 3500 Krems, Austria \\ \{Eva.Mayr, Michael.Smuc, Hanna.Risku\} edonau-uni .ac .at \\ ${ }^{2}$ Department of Information and Knowledge Engineering (ike), Danube University Krems, \\ Dr. Karl Dorrek Str. 30, 3500 Krems, Austria \\ \{Wolfgang.Aigner, Alessio.Bertone, Tim. Lammarsch, \\ Silvia.Miksch\} @donau-uni.ac.at
}

\begin{abstract}
Especially in ill-defined problem spaces, more than one exploration way leads to a solution. But often visual analytics methods do not support the variety of problem solving strategies users might apply. Our study illustrates how knowledge on users' problem solving strategies can be used in the participatory design process to make a visual analytics method more flexible for different user strategies. In order to provide the users a method which functions as a real scaffold it should allow them to choose their own problem solving strategy. Therefore, an important aim for evaluation should be to test the method's flexibility.
\end{abstract}

Keywords: Problem solving strategies, information visualization, visual analytics, evaluation.

\section{Introduction}

"The goal of visual analytics is to create software systems that will support the analytical reasoning process" [19]. Following this rationale, we are currently engaged in a research project which aims to support the daily work processes of business consultants by means of novel visual analytics methods. To ensure that the methods successfully support data exploration, prototypes are iteratively evaluated in real-world settings with real users and refined based on evaluation results.

A successful visual analytics method allows users to generate insights and supports exploratory data analysis. Therefore, evaluation techniques building on task completion time and number of errors were criticized as restricted in the past [2]. In more recent evaluations researchers code and count the insights gained [13][17]. Though insights are an outcome of cognitive processes during exploratory data analysis, they are not directly linked to the task at hand. To understand the users' cognitive processes while they are completing a task (or failing to do so) we proposed to analyze the problem solving processes [10]. Problems are the users' subjective representations of 
an objectively given task [11]. Therefore, analyzing problem solving strategies can help us to understand how individual users approach the tasks in evaluation studies.

To gain meaningful results from evaluations, it was proposed that users have to solve ecologically valid tasks during the evaluation procedure [12]. Therefore, we asked experts to provide not only real-world data sets, but also real-world tasks of different complexity for the evaluation in our research project DisCō. During earlier stages in the participatory design process of two visual analytics methods, we observed that users apply many different strategies to solve these tasks. There was not one single problem solving strategy that led to a correct solution - as many ways lead to Rome, users reached a solution via different paths. Still, some of the strategies that were applied did not yield a sufficient solution. Interestingly, the question of how problem solving strategies interact with characteristics of the method/visualization and task completion was not addressed in prior research.

In this paper we argue that though some users may have taken a wrong path, for others the method probably impeded a successful strategy. In our view a successful information visualization allows for a variety of different problem solving strategies. By analyzing users' problem solving strategies we can understand how a visual analytics method supports or impedes the problem solving processes - better than by coding and counting insights alone. In addition, we can generate ideas how the method should be improved to allow for frequently used problem solving strategies. By looking more deeply into the problem solving processes, the evaluation produces results beyond task completion, number of insights, time, and errors.

To prove our point, we first give some background information on problem solving and discuss how an information visualization or visual analytics method can act as a scaffold. To show how the flexibility of a method can be evaluated and improved we present results from our experimental study.

\section{Problem Solving}

"Research in situated and everyday problem solving (e.g., Lave, 1988) makes clear distinctions between convergent problem-solving thinking and the thinking required to solve everyday problems" [7]. Therefore, it is important to distinguish between different types of problems and identify which problem solving strategies are applied to solve them.

\subsection{Problem Types}

In cognitive psychology, two major types of problems are distinguished [7]: Welldefined problems have one correct solution and provide all information needed to solve them. Typical locating- (e.g., finding a date) or identifying-tasks (e.g., finding the maximum) [20] can be associated with such kind of problems. In contrast, illdefined problems have more than one solution and often include only fragmentary information. Exploratory data analysis only seldom converges in one single correct solution; therefore, it can be classified as ill-defined. 


\subsection{Problem Solving Strategies}

These two types of problems not only differ in the number of correct, respectively plausible solutions, but also in the processes needed to get to a solution. A problem solving strategy is "a technique that may not guarantee solution, but serves as a guide in the problem solving process" [5].

Ill-defined, everyday problems can be solved in different ways, probably leading to different solutions. This is a very creative process [7]. Therefore, it is difficult to predict either solutions or strategies applied for such problems.

People who are able to successfully solve well-defined problems cannot necessarily solve ill-defined problems, too [14]. To solve well-defined problems, one has to know rules and strategies and know when to apply which. For ill-defined problems one has to generate different solutions and evaluate them based on one's own knowledge and opinions.

This could be explained by different kinds of problem solving strategies applied to well-defined and ill-defined problems: In well-defined problems, users are more likely to have a schema (including knowledge on procedures, relevant information, and goals) which can be applied to solve the problem. In contrast to these schemabased problem solving strategies [5], ill-defined problems might require a searchbased problem solving strategy [5] to reach a solution. Users have to search for relevant information, decompose the problem into sub-problems (which can again be more well- or ill-defined), and identify goals. Which strategy is applied, depends to a great extent on the expertise of the user (does he have a schema available?) and the problem at hand.

\subsection{Scaffolding Problem Solving}

The aim of visual analytics is to support the problem solving process [19]. From the view of situated cognition, the visual representations serve as scaffolds [3] for the problem solving process. By visualizing and pre-processing the information, these methods reduce the need to process and store data in memory.

Experts are more suited to solve problems, as they can faster and better identify the type of problem at hand and have a bigger repertoire of problem solving strategies [7]. To serve as real scaffold, the visual analytics method should consequently allow for multiple problem solving strategies to support the creative process of solving illdefined problems at work.

Let us exemplify our point with an example from everyday life: You want to tighten a screw, but do not have a screwdriver at hand. With good skills and strength, you might be able to tighten it with a simple coin, a key, or a pocket knife. But if you are provided a Swiss army knife, you will solve this problem more easily.

To ensure that a visual analytics method is such a flexible scaffold, we evaluated how many different problem solving strategies our method supports and which strategies it impedes.

\section{Identifying Problem Solving Strategies in Participatory Design}

In a study within the research project DisCō we compared two different prototypes, GROOVE [9] and a variant of the Multiscale visualization [16]. Whereas GROOVE 
allows users to interactively fold and unfold time scales, the Multiscale visualization shows all temporal granularities one below the other.

\subsection{The Aims of and Visualizations Used in the Project DisCō}

The project DisCo ${ }^{-1}$ aims at designing novel interactive Visual Analytics methods that support users to discover temporal patterns and their relationships. In doing so, a large number of time-related aspects need to be considered [1]. One aspect that is especially worth exploring is the calendar aspect. The structures of time strongly determine phenomena which can be found in time-oriented data. For instance, the patterns of monthly sales vary strongly due to differences in the arrangement of workdays, weekends, and holidays.

Our target users handle application scenarios of data analysis in different industrial or service sectors (e.g., transportation, call centers, retail, health care) and in the public sector. Users reported that they are often confronted with ill-defined problems [18]. To solve such problems, temporal analysts have to identify temporal patterns of different time granularities in the data. Consequently, our users have to (1) gain an overview of the data set, (2) identify relevant and define specific time granularities (e.g., one business day can last from 6am to $6 \mathrm{pm}$, from midnight to midnight), and (3) find anomalies and relevant patterns, trends, and relations within this data set. To provide visualizations that suit these requirements, we researched various advancements on the basis of pixel-based visualizations.

Pixel-based visualizations [8] use position in two dimensions and color to encode data. For time-oriented data, the position within a two-dimensional grid can be determined by the timestamp of a data element. In such visualizations, it is necessary to find a way to encode the value of single data points, as both axes are used for time granularities. One way is using different colors for different values.

The tripartite Multiscale visualization [16] (see figure 1) is a further development of pixel-based visualizations. It uses a parallel overview of average values as a guidance and information source as well. The daily scale data area shows squares for each month. Each square contains a small pixel-based visualization in itself, arranging the data using week of month and day of week as coordinates. The monthly scale data area shows the same squares filled with a single color based on the monthly average. The yearly scale data area shows only one square per year, with the color based on the yearly average. However, the overview is not optimal as guidance, as it is spatially apart from the details. The eye of the beholder constantly has to jump between the two parts, which is rather a long distance, comprising the danger of mistakes and straining working memory.

We have developed a visualization called GROOVE that contains several advancements compared to the Multiscale visualization [16] [9]. The central advancements are overlay techniques that provide an overview which is integrated with the details more closely and interactive methods for changing the view. For the study described in this paper, we excluded the overlay possibilities in order to get a clear comparison between parallel views and interactive change of detail level. The interactive visualization (see figure 2) we compared to the Multiscale visualization works as follows: Users are shown an overview with large areas coloured based on average

${ }^{1}$ http: / /www. donau-uni.ac.at / disco (accessed on May 4, 2010). 


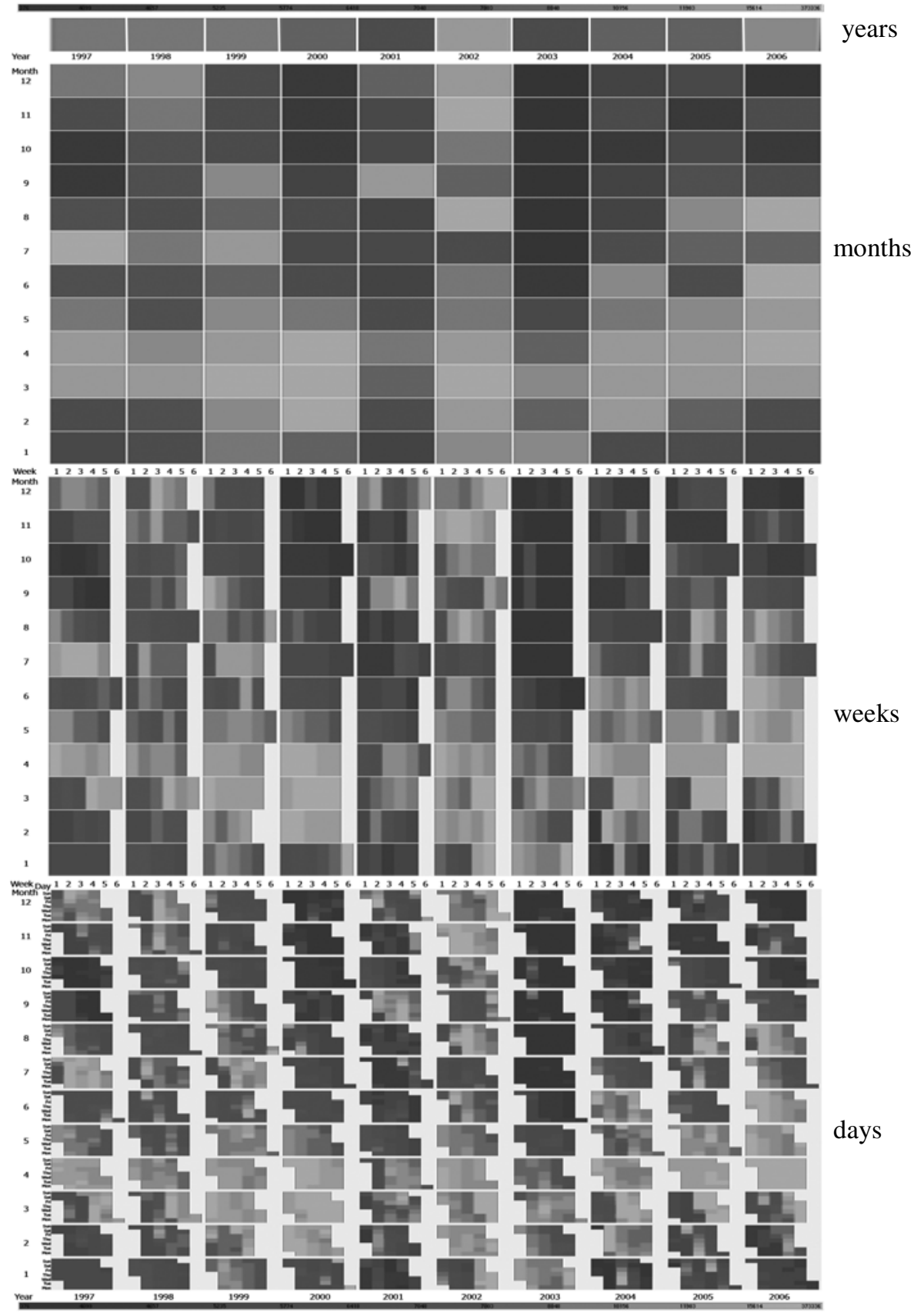

Fig. 1. Multiscale visualization of meteorological data set with yearly, monthly, weekly and daily views 
values. By clicking left on such an area, they can show more details consisting of smaller pixels. By clicking right inside an area, the detail level is reduced. It is also possible to align the detail level for the whole visualization using a double-click.

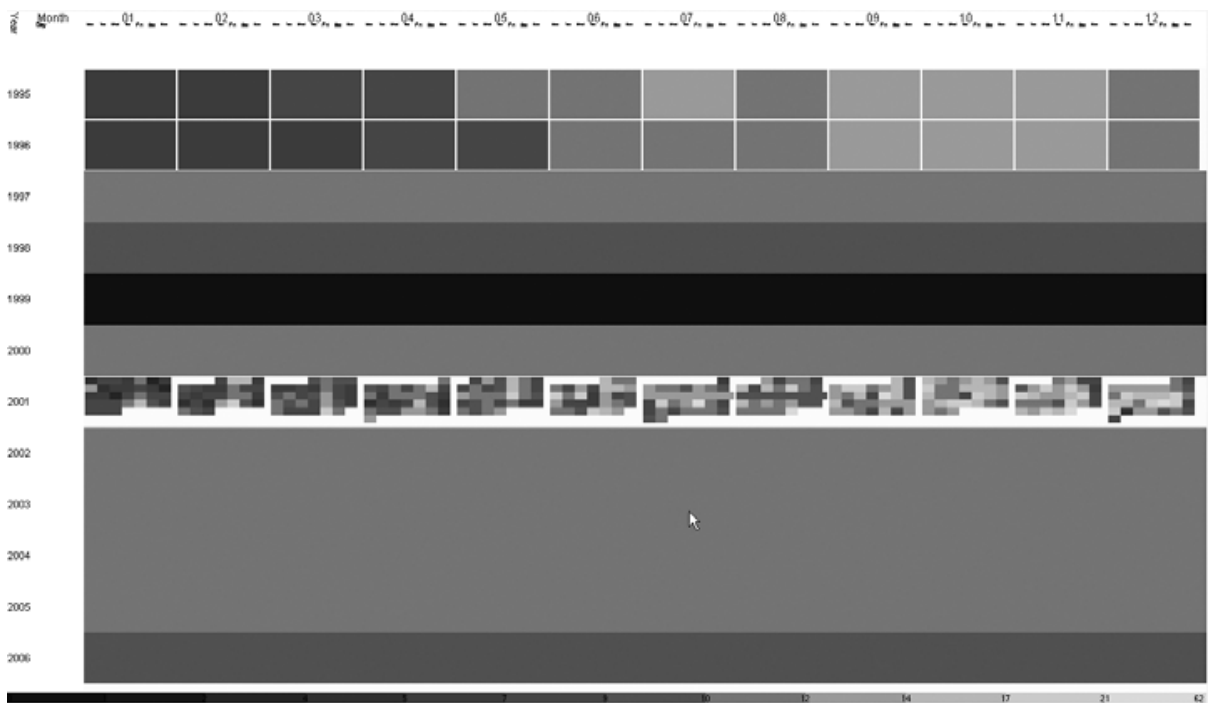

Fig. 2. Our interactive test environment of the traffic dataset: For most of the years, only an average value is shown. For the first two years 1995 and 1996, a more detailed view for the months has been opened. For 2001, average daily values are displayed.

\subsection{Experimental Setting: Material}

We used five different datasets of similar complexity from different domains in our study: meteorology, economy, traffic, education, and finance. The data values are presented in quantiles, with temporal granularities ranging from hours up to decades.

The meteorology dataset shows gauging data from the Traisen, a river in Lower Austria for the time-range of several years. The economy dataset shows turnover data from the business area of gastronomy for one year. The traffic dataset shows traffic accidents of novice drivers in Austria between the ages of 18 and 30 for several years. The education dataset contains total access statistics for infovis-wiki.net, a wiki for Information Visualization which is heavily used for e-learning purposes. Finally, the finance dataset shows the exchange rates of the Euro and the US Dollar over the last two decades. For these datasets, our users had to solve up to seven tasks, for example "What's the quantile value on Christmas Day in 2007?", "Are there clues for errors in the data?", "When looking at separate days, are there several typical trends over the hours of day?", or "Which global and local trend can you identify?".

\subsection{Experimental Setting: Participants}

Twelve people who are experienced in the exploration and analysis of time-oriented business data participated in our study. They had to solve the above mentioned welland ill-defined problems with five temporal data sets (one for familiarisation, two 
using GROOVE, two using Multiscale visualizations, compare table 1). Participants were randomly assigned to versions $\mathrm{A}$ and B. For each data set, users solved two general tasks and a set of three to five domain-specific tasks.

Table 1. Research design

\begin{tabular}{lccccc}
\hline Data Set & meterology & economy & traffic & education & finance \\
\hline Method Version A & GROOVE & GROOVE & GROOVE & Multiscale & Multiscale \\
Method Version B & Multiscale & Multiscale & Multiscale & GROOVE & GROOVE \\
\hline
\end{tabular}

A problem solving process includes different cognitive [7] and perceptual [6] processes. Therefore, we used multiple process measures to study the participants' problem solving strategies. We logged participants' interaction with the method, tracked their eye movements, observed their behaviour, and asked them to think aloud during the experiment [17]. We integrated these data sources, segmented them according to the tasks, and documented the users' strategies and success levels.

In the context of data visualizations and visual analytics methods, three levels of graph comprehension can be differentiated [4]: (1) reading the data (i.e. extracting data, locating), (2) reading between the data (i.e. finding relationships, integrating), and (3) reading beyond the data (i.e. extrapolating from the data, generating). Welldefined problems require level 1 and sometimes level 2, whereas ill-defined problems require all three levels to be solved successfully. In the following we will present two exemplary problems - one from level 1 , reading the data, and one from level 3 , reading beyond the data.

\subsection{Well Defined Problem: Extracting a Concrete Value}

For each data set and method, our users had the same task to solve: to name the data value on Christmas day in a concrete year (the other tasks compare Section 3.2). This is a rather narrow and well-defined task, as it has a single correct solution. But despite this fact, we observed a variety of different strategies that were applied.

Two excerpts from one participant shall illustrate the variance of strategies:

Economy data set (Groove):

"Christmas Day 2007, okay. [...] now I'll try to find Christmas. Oh, there are weeks, not month. I have to calculate back. It has to be 51 or 52. [...] Oh, when Monday ends earlier on this day, it has to be Christmas. [...] And I assume that this orange is somewhere in the range of $365 . "$

Finance data set (Multiscale visualization):

"Christmas Day 2007. There is 2007. Mostly it's the end of the year. Okay I assume this is the 31st of December. Actually it does not matter, somewhere here. Everything is the same colour, and it's the darkest. [...] about 1.36, okay."

Problem Solving Strategies. To solve this problem, users had to identify the location of this date and to associate a value to the data point. We analyzed the different process measures described above and observed seven problem solving strategies which were applied either individually or in combination with each other: (1) count days from the beginning of December or (2) from the end of December; (3) map specific 
data characteristics (e.g., shop closes earlier on 1 day, less activity) onto the characteristics of Christmas day (see the economy excerpt above for an example); (4) use external scaffold (e.g., calendar on mobile phone) to determine the associated day of week; (5) remember the correct day of week from a prior dataset; (6) approximate the location by searching for week 51 ; (7) estimate roughly (see the finance excerpt above for an example).

The strategies applied differed highly between participants, but also within participants. No one used one single problem solving strategy consistently. A more detailed look at the variations showed that participants applied problem solving strategies differently in dependence of the method and the data set at hand (see figure 3 ).

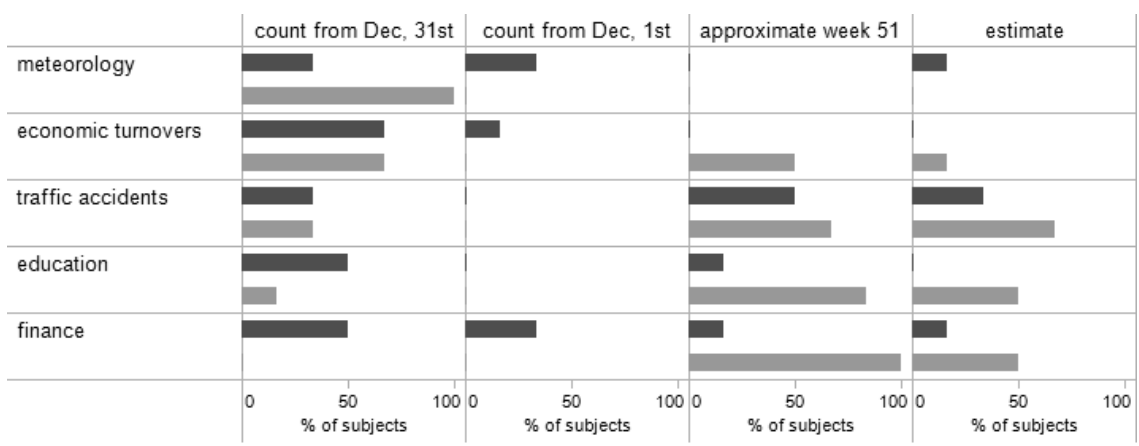

Visualization

Groove

Multiscale

Fig. 3. Percentage of users applying different problem solving strategies for 5 data sets

Obviously some data sets suggest specific strategies. For example, the financial data set had only little variance within weeks. Therefore, approximating the location and roughly estimating the correct value was a highly efficient strategy, leading to correct solutions in $82 \%$ of all cases. The economic turnover data set, on the other hand, was only solved correctly by $17 \%$ of the participants. It has high variance within the data and is visualized on a weekly rather than on a monthly basis. Therefore, only participants who counted from the end of the year solved this problem. Every fourth participant was not able to generate any solution at all.

A clear difference exists also between the two methods, GROOVE and Multiscale visualization, in the problem solving strategies applied. This can be seen clearly in the traffic accidents data set, where all Multiscale visualization users counted from the end of the year, whereas the GROOVE users applied a variety of problem solving strategies. This difference also results in different solution probabilities: When participants used the GROOVE, they solved the task in $50 \%$ of the time; whereas when they used the Multiscale visualization, only $27 \%$ solved the task. With the Multiscale visualization, they often experienced problems to find the data point (33\%), but also for the second step in solving the problem: to differentiate between colours $(10 \%)$ and to associate the colour to the scale $(10 \%)$. 
Design Implications. To improve the two methods, participants made some remarks which can be turned into suggestions for improvements directly:

- $\quad$ Labelling the figure on both sides

- Showing date and value on mouse over

- Ease making the association of data points with the legend

Besides, many users experienced problems to locate the Christmas day. Therefore, the methods should be improved, for example, by providing a tooltip or a search function for specific dates (e.g., with a calendar overlay).

With the Multiscale visualization, users experienced many problems in identifying a specific data point and in differentiating between the colours used. This problem could be solved by providing an optical zoom function and (a user-customized) colour scale to increase the contrast for specific scale segments.

\subsection{Ill Defined Problem: Drawing an Inference from the Data}

For the economy data set, participants were asked from which gastronomic business these data are from (e.g., snack restaurant, bakery, or coffee house). This is a clearly ill-defined problem as it allows for different plausible answers (even though the data stem from one concrete business) and as a wide range of information sources is relevant. To make this conclusion, users had to build on the different features of the visualization and draw inferences from their insights. They had to identify patterns and compare them to patterns from their own prior knowledge, raise hypotheses and test them against the patterns found.

An excerpt from one participant (female, Multiscale visualization, implausible solution) shall illustrate the nature of this task. She does identify the daily and weekly sales patterns, but ignores the opening hours, the annual sales pattern, and the amount of turnover.

"They sell a lot in the afternoon, mostly. Which kind of business? Nonsense, it's not afternoon, but rather evening. I would say: after-work. But also in the morning. I would say a café. No it's too late for a café. They are already eating. But also earlier, about 10 am or 9 there is a lot. That's strange, it does not fit. Evening restaurant. Especially on Saturday there is nearly anyone there. Yes, it's a café-restaurant."

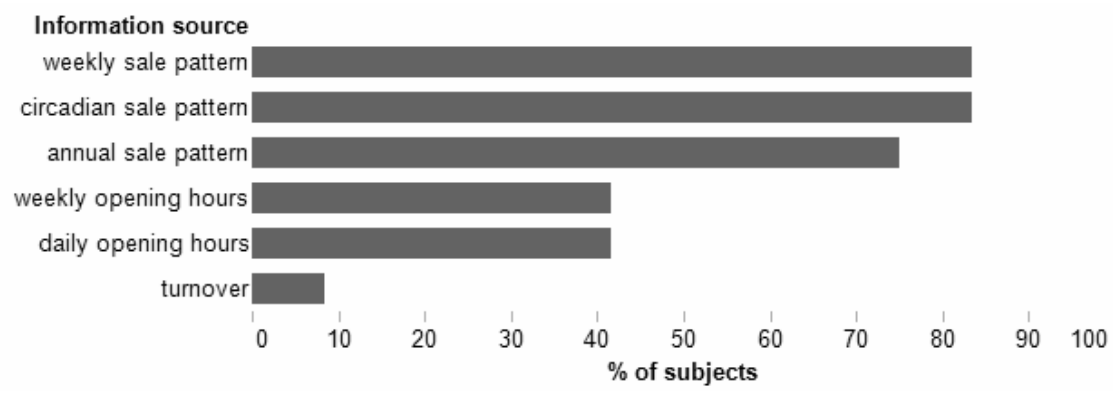

Fig. 4. Percentage of participants who used a specific information source 
Problem Solving Strategies. For this very open task, users relied on six different information sources to infer the problem solution (see figure 4): Whereas the sale patterns (circadian, weekly, and annual) were used by most participants, only some also took the opening hours (weekly and daily) into account. Only one participant considered the amount of the turnover as a relevant information source.

No difference existed between the two methods, GROOVE and Multiscale visualization, in the information sources used and the quality of the solution gained. Overall, $17 \%$ of the users were not able to generate any solution for this task. Half of the remaining participants generated a plausible, near-to-correct solution (42\%), the other half no plausible solution (42\%).

We compared the problem solving strategies used by these three groups and found that the quality of the solution correlated with the number of information sources participants took into account (see figure 5): If they considered only two or three different kinds of information they were likely to generate a wrong solution. If they considered three to four information sources, they did not generate any information ("I give up. I've no idea what this could be."). Only if they considered a higher number of more than four different information sources, were they likely to generate a plausible, correct solution.

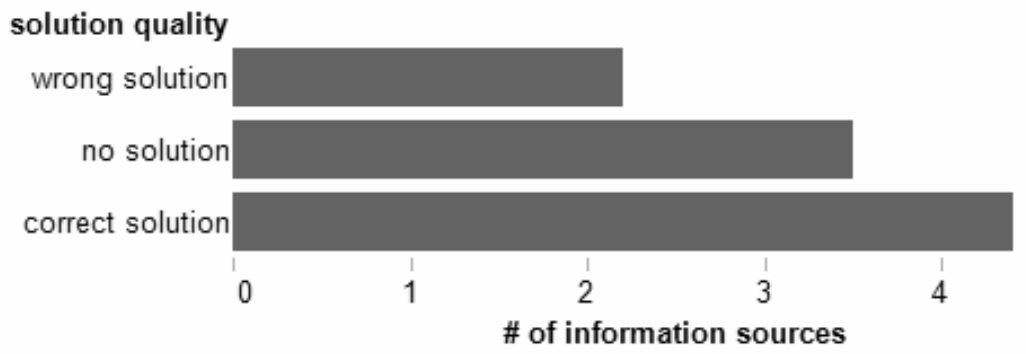

Fig. 5. Number of information sources used in dependence of the solution quality

When we look at these kinds of information more qualitatively, we see a tendency that those participants who considered the weekly and daily opening hours were more likely to come to a correct solution. A frequent wrong solution neglected the information that the business closed before $8 \mathrm{pm}$ and, therefore, could not be a dinner restaurant.

The difference cannot be explained by a motivational deficit as participants took a similar amount of time, independent from the quality of their solution (correct: 3.1 min, incorrect: $2.1 \mathrm{~min}$ ). Only those, who did not come to a solution at all, took more time $(8.3 \mathrm{~min})$.

Design Implications. A crucial factor to generate a plausible solution for this task is to take into account not only the temporal patterns of the data set, but also the temporal boundaries of the visualization. Many participants failed because they did not take the daily and weekly opening hours into account. To make the daily opening hours more salient, one could highlight the closing hours by showing not only labels for those hours of the day where data exist, but also for those where no data exist. Another possibility would be to increase the size of the labels. 
Another question (that remains to be solved) is how participants can be encouraged to test their hypotheses against more information sources and thereby become more likely to discover wrong assumptions.

A possibility within the GROOVE would be to lead users through all granularities step by step and thereby make it easier to check possible solutions against all temporal granularities. A disadvantage of such a solution is that the user looses freedom of action.

\section{Conclusions}

In this paper we presented two examples of how users' problem solving strategies can be analyzed and how this knowledge be used to improve a visual analytics method during the participatory design process. For the well-defined problem (finding a date and naming the associated value) users applied various strategies. We observed that for different data sets different problem solving strategies led to a correct solution. Similarly the two visual analytics methods afforded different strategies - and different success rates. But there was no single strategy, nor a combination of strategies that always led to a correct solution.

For the ill-defined problem we identified a concrete information source as necessary prerequisite to generate a plausible solution. In ill-defined problem solving, it seems to be important that users do not abort the search-based problem solving process too early, but engage in multiple rounds of generating and testing hypotheses against the data at hand.

Our results confirm that the best problem solving strategy varies from data set to data set and from visual analytics method to method. Especially expert users are more likely to select the most appropriate strategies for the situation at hand [15]. Because experts are more likely to adjust their strategies and use them more flexibly, a visual analytics method should enable the application of a variety of problem solving strategies. Even within the short time frame of the experiment (1 to 1.5 hours) we observed that some users adjusted their problem solving strategies to the possibilities provided by the visual analytics method. Such adaptive behaviour is even more likely when the visual analytics method is used over a longer time period (even though methodspecific schemata for problem solving are likely to be built up over time as well). Despite the possibility to open new ways to solve a problem, the method should not impede established and successful problem solving strategies. Therefore, we propose to let users cut their own path and provide them with the visual analytics methods required to find their own way to the problem's solution.

In the field of information visualization and visual analytics, different assumptions exist concerning the effectiveness of a visualization [1][12][13][19]. We argue that an effective information visualization or visual analytics method allows for multiple ways to solve a problem. Our results confirm that different problem solving strategies are applied in dependence of the user's expertise, the task, the data set, and the method at hand. To develop a successful visual analytics method, users should be allowed to choose between different ways to the problem's solution. In the participatory design process of novel visual analytics (but also other) methods, we can enhance a method by analyzing the users' problem solving strategies and by mapping them closely in the next design phase. 


\section{Acknowledgements}

This research was conducted within the DisCō research project, supported by the program "FIT-IT Visual Computing" of the Federal Ministry of Transport, Innovation and Technology, Austria (Project number: 813388).

\section{References}

1. Aigner, W., Miksch, S., Müller, W., Schumann, H., Tominski, C.: Visual Methods for Analyzing Time-Oriented Data. IEEE T. Vis. Comput. Gr. 14, 47-60 (2008)

2. Bertini, E., Perer, A., Plaisant, C., Santucci, G.: BELIV 2008: Beyond time and errors novel evaluation methods for information visualization. In: Proceedings of BELIV 2008, pp. 3913-3916. ACM Press, New York (2008)

3. Clark, A.: Being There. Putting Brain, Body, and World Together Again. The MIT Press, Cambridge (1997)

4. Friel, S.N., Curcio, F.R., Bright, G.W.: Making sense of graphs: Critical factors influencing comprehension and instructional implications. J. Res. Math. Educ. 32, 124-158 (2001)

5. Gick, M.L.: Problem-solving strategies. Educ. Psychol. 21, 99-120 (1986)

6. Grant, E.R., Spivey, M.J.: Eye movements and problem solving: Guiding attention guides thought. Psychol. Sci. 14, 462-466 (2003)

7. Jonassen, D.H.: Instructional design models for well-structured and ill-structured problem solving learning. Educ. Tech. Res. 45, 65-94 (1997)

8. Keim, D., Kriegel, H.-P., Ankerst, M.: Recursive Pattern: A Technique for Visualizing Very Large Amounts of Data. In: Proceedings of IEEE Visualization (Vis 1995), pp. 279$286(1995)$

9. Lammarsch, T., Aigner, W., Bertone, A., Gärtner, J., Mayr, E., Miksch, S., Smuc, M.: Hierarchical Temporal Patterns and Interactive Aggregated Views for Pixel-based Visualizations. In: Proceedings of IV 2009, pp. 44-49. IEEE Computer Society Press, Los Alamitos (2009)

10. Mayr, E., Smuc, M., Risku, H.: Many Roads Lead to Rome. Mapping Users' Problem Solving Strategies. In: Proceedings of BELIV 2010. ACM Press, New York (2010)

11. Newell, A., Shaw, J.C., Simon, H.A.: Elements of a theory of human problem solving. Psychol. Rev. 65, 151-166 (1958)

12. Robertson, G.: Beyond time and errors - position statement. In: BELIV 2008 (2008), http://www.dis.uniroma1.it/ beliv08/pospap/robertson.pdf

13. Sarayaia, P.B., North, C., Duca, K.: An insight-based methodology for evaluating bioinformatics visualizations. IEEE Trans. Vis. Comput. Graph 11, 443-456 (2005)

14. Schraw, G., Dunkle, M.E., Bendixen, L.D.: Cognitive processes in well-defined and illdefined problem solving. Appl. Cognitive Psych. 9, 523-538 (1995)

15. Schunn, C.D., McGregor, M.U., Saner, L.D.: Expertise in ill-defined problem solving domains as effective strategy use. Mem. Cognition 33, 1377-1387 (2005)

16. Shimabukuro, M., Flores, E.F., de Oliveira, M.C.F., Levkowitz, H.: Coordinated views to assist exploration of spatio-temporal data: A case study. In: Proceedings of the 2nd International Conference on Coordinated and Multiple Views in Exploratory Visualization (CMV 2004), pp. 107-117. IEEE CS Press, Los Alamitos (2004)

17. Smuc, M., Mayr, E., Lammarsch, T., Aigner, W., Miksch, S., Gärtner, J.: To score or not to score? Tripling insights for participatory design. IEEE Comput. Graph. 29(3), 29-38 (2009) 
18. Smuc, M., Mayr, E., Lammarsch, T., Bertone, A., Aigner, W., Risku, H., Miksch, S.: Visualizations at first sight. Do insights require training? In: Holzinger, A. (ed.) HCI and Usability for Education and Work, pp. 261-280. Springer, Berlin (2008)

19. Thomas, J.J., Cook, K.A.: Illuminating the Path: The Research and Development Agenda for Visual Analytics. IEEE Computer Society Press, Los Alamitos (2005)

20. Valiati, E.R.A., Pimenta, M.S., Freitas, C.M.D.S.: A taxonomy of tasks for guiding the evaluation of multidimensional visualizations. In: Proceedings of BELIV 2006, pp. 1-6. ACM Press, New York (2006),

http: //doi.acm.org/10.1145/1168149.1168169 